distance from $B_{j}$ to $B$ is equal to $1 / j$. Let $W$ denote the set of intervals composed of all the intervals of $Q$ and all intervals $t$ such that $t$ is equal to some segment of $G$ plus its endpoints. Let $F$ denote the point set $B+K+K_{1}+K_{2}+K_{3}+$. . . . It can be seen that $F$ is a closed point set of measure zero and that it is covered in the Vitali sense by the set of intervals $W$. But $W$ contains no subset $\bar{W}$ such that $\bar{W}$ covers $F$ and such that the sum of the lengths of the intervals of $\bar{W}$ exists (as a finite number).

The University of Texas

\title{
THE CONVERGENCE OF A GENERAL MEAN OF MEASUREMENTS TO THE TRUE VALUE*
}

\author{
BY E. L. DODD
}

Theorem. For positive measurements $x_{i}$ suppose that the frequency function $\phi(x)$ has a positive lower bound $b$ in some interval $(a-k, a+k)$ about the true value $a$, and that for each positive $r$

$$
m_{r}=\int_{0}^{\infty} x^{r} \phi(x) d x, \quad(\text { finite }) ; \quad m_{0}=1 .
$$

Then there exists a continuous increasing function $f(x)$, with inverse $f^{-1}$, such that if $\epsilon>0, \eta>0$, and

(2) $M=f^{-1}\left\{\right.$ Arithmetic mean of $\left.f\left(x_{i}\right)\right\}, i=1,2, \cdots, n$, then, when $n>$ some $n^{\prime}$, there is a probability $>1-\eta$ that

$$
|M-a|<\epsilon \text {. }
$$

Proof. For (3) the special condition $\dagger$ is

$$
f(a)=\int_{0}^{\infty} f(x) \phi(x) d x .
$$

* Presented to the Society, February 27, 1926.

$\dagger$ Dodd, Functions of measurements under general laws of error, SkandNavisk AkTUaRietidskRift, vol. 5 (1922), pp. 133-158. Apply theorem, p. 135, taking $\Sigma f\left(x_{i}\right)<n f(a+\epsilon)=n(1+\delta) f(a)=z$; then $\Sigma f\left(x_{i}\right)<n f(a-\epsilon)$. 
If $f(x)=x^{r}$, (4) requires that $a^{r}=m_{r}$. This can be satisfied if $m_{r}<a^{r}$ for some $r$; since $m_{r}$ is a continuous function of $r$, and if $r$ is large enough,

$$
m_{r} \geqq b \int_{a}^{a+k} x^{r} d x>a^{r} .
$$

In any case, however, (4) can be satisfied by

$$
f(x)=c x^{r}+z ; \quad z=2 x-x^{2} / a, \quad x \leqq a ; \quad z=a, x \geqq a,
$$

because a positive $c$ can be found satisfying $c a^{r}+a=c m_{r}+g$, where $g<a$, noting that $z \leqq \alpha$, and $z<a$ inside $(a-k, a)$ where $\phi(x) \geqq b$.

This $M$ satisfying (3) reduces to the arithmetic mean only if $m_{1}=a$.

The University of Texas

\section{CONSECUTIVE QUADRATIC RESIDUES*}

\section{BY A. A. BENNETT}

By an extension of the methods described in a paper to appear shortly in the TôHOKU MATHEMATICAL JOURNAL, I have succeeded in proving that for each prime greater than 193 there is at least one sequence of five consecutive positive reduced quadratic residues. The proof entails the examination of many hundred linear forms which together include all primes. Since the method would prove excessively laborious for even the next case, that of six consecutive quadratic residues, the computational details seem hardly to warrant the space required for their complete publication. As a result of the actual construction of a complete table of quadratic residues for all primes less than 331, we obtain the brief table subjoined. Here $p$ denotes in turn each prime number, $r$ denotes for the given $p$ the maximum number of positive reduced quadratic residues which a ppear

*Presented to the Society, October 31, 1925. 